\title{
NOTA SOBRE LA BIOLOGIA DE LOS DEPOSITOS FANGOSOS CIRCALITORALES FRENTE A PUNTA DEL ESTE, URUGUAY
}

\author{
MARIO A. CACHÉS \\ Museo de Historia Natural de La Paloma, Uruguay
}

\section{SYNOPSIS}

A circalltoral muddy bottom community is studied off Punta del Este, Uruguay. From this study a relevant fact is observed: the coexistence between deposit-feeders and suspension-feeders. It has long been thought that they are excludent trophic groups. The coexistence may be explained by the deposit stability favourished by a dense population of the tube-building polychaete Onuphis setosa.

\section{Introducción}

De tiempo atrás la literatura especializada ha venido acumulando datos que indican que las poblaciones de organismos bentónicos detritívoros y las de suspensívoros tienden a excluirse. Rhoads \& Young (1970) introdujeron el término "amensalismo de grupo trófico" para denominar a este fenómeno de exclusión. Ambos autores muestran, además, que la segregación espacial de estos grupos es común en todo el mundo.

Es en atención a estos antecedentes que consideramos particularmente interesantes una serie de muestras bentónicas, obtenidas frente a Punta del Este, en las cuales se constata que ambos coexisten.

Las muestras a que hacemos referencia fueron obtenidas durante la campaña de prospección pesquera "Striker", en los afios 1971 y 1972. Una reseña sobre esta campafía ha sido publicada por Amaro (1974).

\section{El Area}

El área estudiada (Fig. 1) corresponde a un fondo fangoso de 20 a $30 \mathrm{~m}$ de profundidad. Este fondo resulta de la deposición de los limos y arcillas transportados en suspensíón por las aguas del Río de la Plata (Ottmann \& Urien, 1965, 1966). La influencia del río mencionado hace que el promedio de salinidad superficial registrado en Punta del Este sea de $24,8 \%$ o. La temperatura superficial fluctúa entre 17 y $24^{\circ} \mathrm{C}$ para el mes más cálido (Febrero) y 8 y $12^{\circ} \mathrm{C}$ para el mes más frío (Agosto).

Por otra parte la elevada turbidez impide el desarrollo vegetal más allá de los $10 \mathrm{~m}$ de profundidad, de modo que los depósitos fangosos en estudio pueden considerarse pertenecientes al $\mathrm{Pi}$ so Circalitoral.

\section{Material y Métodos}

Las muestras bentónicas se obtuvieron con una rastra convencional de boca triangular. Complementariamente se uso un "beam trawl". Empleando la rastra se tomó un total de 9 muestras, de hasta $5 \times$ del sedimento cada una, distribuídas al azar dentro del área estudiada. El sedimento se trió con malla de $1 \mathrm{~mm}$ de pase y el material resultante, fijado en formol $10 \%$, quedó depositado en el Instituto de Investigaciones Pesqueras de Montevideo.

\section{Resultados}

La composición de las muestras, que es marcadamente homogenea en toda el área, puede resumirse de la siguiente forma, tomando en cuenta la modalidad alimentaria de los organismos que las integran:

Organismos detritívoros: Onuphis setosa (dominante; representado en todas las muestras con el mayor número de individuos); Praxileilla sp. (abundante (a); presente en la mayoría de las muestras con numerosos especímenes); Nucula puelcha (a); Macoma urugua. yensis (a); Malletia cumingii (a); Adrana patagonica (raro (r); sólo en alsunas muestras con escasos eiemplares); Pectinaria sp. (r).

Organismos suspensivoros: Pitar rostratus (a); Corbula pata. gonica (a); Ascidiecea indet. (a), fijada a objetos sólidos a nivel del sustrato; Ceriantharia indet. ( $\mathrm{r}$ ).

Organismos omnívoros (potencialmente necrófagos o preda. dores): Hymenopenaeus milleri (a); Artemesia longinaris (a); Leu. rocyclus tuberculosus $(\mathrm{r})$.

Organismos cuya forma de alimentación no se determinó: Sipunculida indet. (a), habitando conchillas vacías de gasterópodos: Periploma compressa $(\mathrm{r})$; Marginella martini $(\mathrm{r})$.
A esta lista deben agregarse varias especies de peces planos que integran la cadena trófica bentónica-demersal, fundamentalmente Pleuronectiformes: Paralichtys, Solea etc.

\section{Discusión}

Al explicar el mecanismo de exclusión Rhoads \& Young (1970, 1971), Young (1971) y Young \& Rhoads (1971), formulan la hipótesis de que es la remoción y resuspensión del sustrato por parte de los detritívoros la que excluye a los suspensívoros. Esta remoción provoca la inestabilidad del depósito por: a) alta tasa de sedimentación debida a la deposición del sedimento que ha sido resuspendido; b) formación de una capa de turbidez a nivel del fondo; c) producción de gradientes de textura y composición en el sedimento. En estos fondos inestables los mecanismos de filtración de los suspensívoros resultan obstruidos. Adicionalmente se ve dificultado el reclutamiento de las larvas de estos organismos.

Basándose en sus observaciones Rhoads \& Young (1970) predicen la existencia de tres formas de agrupamiento de acuerdo a la modalidad trófica y a las condiciones del medio: 1) agrupamiento homogeneo de suspensívoros, donde los detritívoros están ausentes debido a una inadecuada fuente de alimentos; 2) agrupamiento homogeneo de detritívoros, del que han sido excluidos los suspensívoros por el mecanismo de remoción y resuspensión ya considerado; 3) agrupamiento donde coexisten ambos grupos debido a la estabilidad del fondo. Estas predicciones se ven apoyadas por los trabajos de Sandres $(1958,1960)$.

En el caso de Punta del Este, consideramos que la coexistencia observada corresponde a un agrupamiento del tipo 3 . La estabilidad del sustrato estaría determinada por la presencia de una población densa de poliquetos sedentarios, fundamentalmente el dominante, Onuphis setosa. Fager (1964) mostró el papel preponderante que juegan estos organismos en la fijación del devósito

Por otra parte esta comunidad (*) forma énclaves en los depósitos fangosos de áreas costeras protegidas. Así la hemos hallado en el canal de acceso al Puerto de La Paloma (Cachés \& De Fílippo, no publ.). El estudio directo, mediante buceo autónomo, puso de manifiesto que no existe aquí segregación espacial a nivel microtopográfico, como ha sido descrita en otros casos (Rhoads \& Young, 1970). Mostró, en cambio, que la densidad de los poliquetos sedentários, en conjunto, supera los 1000 ind. $/ \mathrm{m}^{2}$, lo que confirmaría la conclusión ya adelantada.

Finalmente seffalemos que durante la campaña "Striker", ahora en las proximidades de Cabo Polonio, $120 \mathrm{~km}$ al N de Punta del Este, y a $20 \mathrm{~m}$ de profundidad, obtuvimos muestras similares a las descritas, lo que indicaría que estamos frente a una comunidad ampliamente distribuída sobre nuestra plataforma.

\section{Agradecimientos}

Agradezco al Dr. Jorge Amaro Padilha la generosidad de su dedicación a lo largo de la campaña "Striker". Asimismo al Arq. Jorge A. De Filippo por su estímulo y apoyo que no saben de desmayos.

\section{Referencias Bibliográficas}

AMARO, J. 1974. Boln Commn nac. Oceanogr. Montevideo, 1: $15-18$.

FAGER, E. W. 1964. Marine sediments: effects of a tube-building polychaete. Science, Wash., 143 (3604):356-359.

(*) El término "comunidad" es empleado con el sentido que le da Sanders (1960): " ... a group of species that show a high degree of association by tending to reoccur together". 
OTTMANN, F. \& URIEN, C. M. 1965. Observaciones preliminares sobre la distribución de los sedimentos en la zona externa del Rio de La Plata. Anais Acad. bras. Ciênc., 37 (supl.): 283-289.

\section{8:209-224.}

1966. Revue Géogr. phỳs. Géol. dyn.,

kHOADS, D. C. \& YOUNG, D. K. 1970. ine influence of deposit-feeding organisms on sediments stability and community trophic structure, J. mar. Res., 28 (2): 150-178.

- - - \& - - 1971. Animal-sediment relations in Cape Cod Bay, Massachusetts. II. Reworking by Molpadia oolitica (Holothuroidea).' Mar. Biol., 11:255-261. re of the softbottom community. Limnol. Uceanogr., $s$
$138-153$,

SANDERS, H. L. 1958. Benthic studies in Buzzards Bay. I. Animalsediment relationships. Limnol Oceanogr., 3:245-258.

- 1960. Benthic studies in Buzzards Bay. II. The structu-

YOUNG, D. K. 1971. Effects of infauna on the sediment and seston of a sub-tidal envíronment. Vie Milieu, suopl. 22, 2:557-571.

------ \& RHOADS, D. C. 1971. Animalsediment relations in Cape Cod Bay, Massachusetts. I. A transect study. Mar. Biol., 11:242-254.

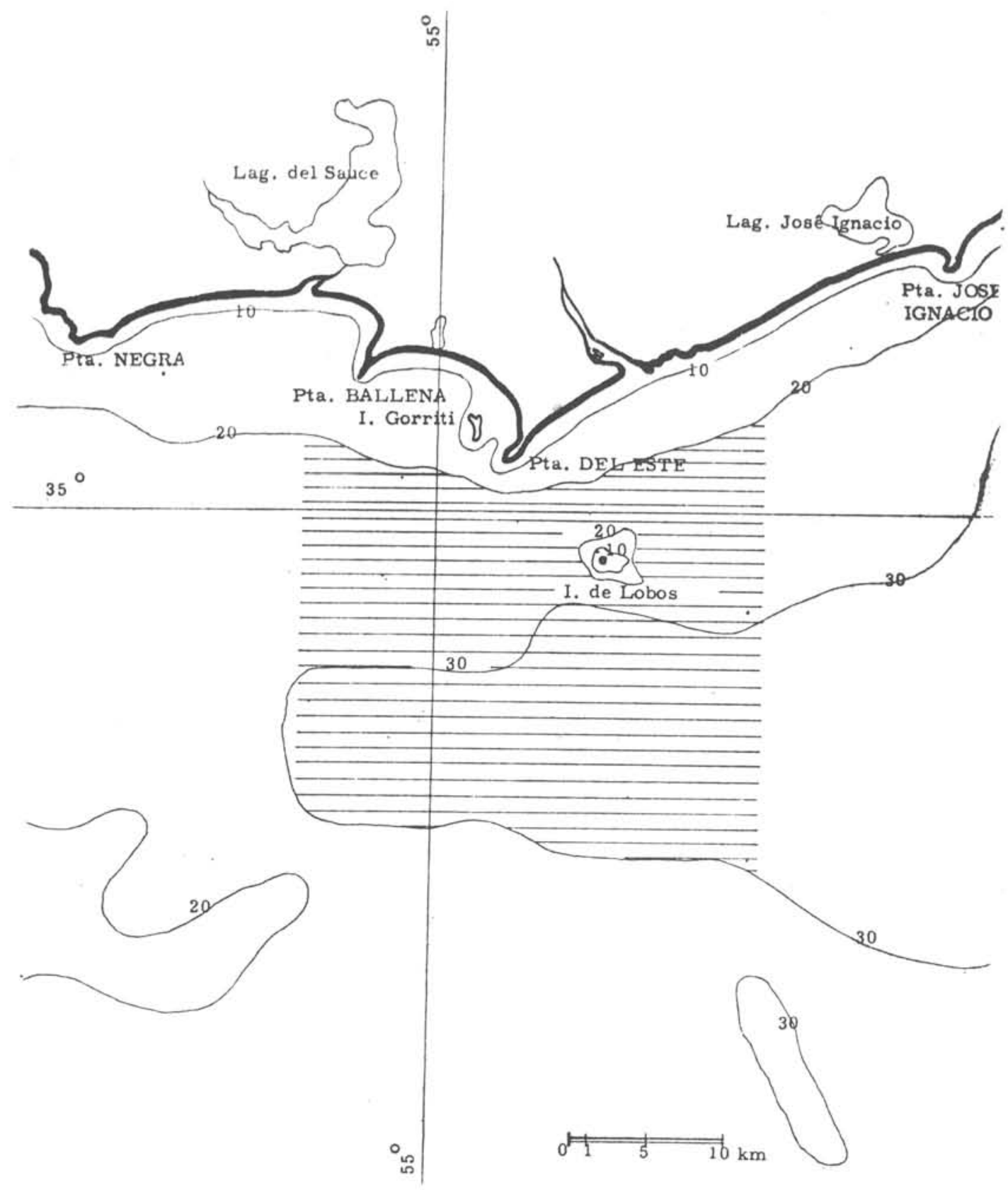

Fig. 1-Punta del Este, en sombreado: área estudiada. Isóbatas en m. 